\title{
Inter carrier interference removal in MIMO-OFDM system
}

\author{
${ }^{1}$ Jasdeep Singh, ${ }^{2}$ Komal Arora \\ (Student, School of Electronics and Communication, Lovely Professional University, Phagwara, Punjab, India) \\ (Assistant Professor, Department of Electronics and Communication, Lovely Professional University, \\ Phagwara, Punjab, India)
}

\begin{abstract}
This review paper is about the schemes of removing the inter carrier interference in the multiple input multiple output-orthogonal frequency division multiplexing system. The inter carrier interference occurs by the frequency offsets. In the cellular communication system the inter carrier interference arises due to the fully loaded traffic conditions at the cell edge .which is removed by the combining the multiple cell coordinated or frequency reuse. The inter carrier interference occurs when the cyclic prefix length lesser than the channel impulse response .To mitigate this interference use the spatial diversity techniques are used. In this method by using the spatial techniques the length of the cyclic prefix becomes more than the channel impulse response. The inter carrier interference can also be removed by the single input multiple output but this is not the better method to remove the inter carrier interference as it does not remove the inter carrier interference properly. Inter carrier interference arises when the wireless channel estimation is done with the help of Doppler spread information and the data symbols detector. In the wireless channel estimation the inter carrier interference is removed by the parallel interference cancellation and the decision statistically combining method.The parallel interference cancellation and decision statistically combining methods are used at the receiver end.
\end{abstract}

Keywords: Ici, Mimo, Mimo-Ofdm, Ofdm, Pic.

\section{Introduction}

The system which uses the multiple antennas both at the transmitter and the receiver side is known as multiple inputs multiple output (MIMO) system. MIMO takes the advantage of the spatial diversity that is obtained by the spatial separation between the antennas in a dense multipath scattering environment. Multiple inputs multiple output system is capable of delivering higher data rates when we employ transmit beam forming and the user scheduling. In the MIMO-OFDM is affected by the interference which is called the Inter carrier interference (ICI).which is removed by the various techniques. There are various ICI removal techniques available i.e.name of techniques are beam forming[1],Space time frequency block codes[2], Combining multiple cells coordinated setting up for downlink[3], Zero padded symmetric conjugate[4],Complex optimum block adaptive method[5],Parallel interference cancellation[6],Curve fitting or weighting factor[7], Turbo equalization algorithm[8],iterative joint estimation[9],Orthogonal space time block code[10],Time domain filtering[11],Minimum mean square error equalization[12].

\section{Block diagram of MIMO-OFDM System}

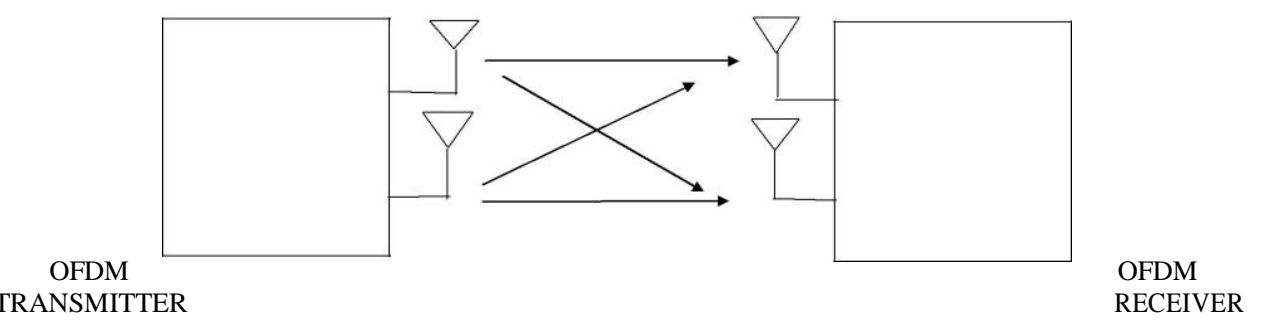

Multiple inputs and multiple output system take the advantages of the spatial diversity that is obtained by the spatial separation between the antennas in an active multiple scattering environments. MIMO system capable of delivering the higher data rate. Orthogonal frequency division multiplexing system divides the spectrum into the sub carriers which the sub carriers placed closed to each other due to orthogonality. MIMO-OFDM system used the multiple antennas at the transmitter and at the receiver side. The spectrum is divided into the super carriers. Each subcarrier has their own particular frequency. Due to the orthogonality .but when the sampling of the sub carrier is done then there is the loss of the orthogonality due to the frequency shifting .which is also called the Doppler shift then their inter carrier interference arises . 


\section{Formula for ICI}

$$
\begin{aligned}
& C I R=\frac{|-S(-1)+2 S(0)-S(1)|^{2}}{\sum_{l=2,4,6}^{N-1}|-S(l-1)+2 S(l)-S(l+1)|^{2}} \\
& S(l-k)=\frac{\sin (\pi(l+\epsilon-k))}{N \sin (\pi(l+\epsilon-k) / N)} \exp \left(j \pi\left(1-\frac{1}{N}\right)(l+\epsilon-k)\right)
\end{aligned}
$$

\section{Beam forming}

\section{ICI Removal Techniques}

It has been noticed that the orthogonal frequency division multiplexing system performance degrades when the channel impulse response becomes more than the length of cyclic prefix. This disturbance is caused by the inter carrier interference which is essentially occurs due to unnecessary multipath delay. Commonly multiple input multiple output beam forming technique used to remove the inter carrier interference .Because multiple input multiple output spatially repress the several of the multipath. Orthogonal frequency division multiplexing scheme is used to diminish the transmission impairments caused by the frequency-selective character of wireless channels. The successful tool need the cyclic prefix length more than the channel impulse response. The extra overhead thus incurred can reduce the spectral efficiency of orthogonal frequency division multiplexing and all the further outdoor systems with large delay spreads. These values were initially planned for interior environments with tiny to modest standards of interruption around the CP length.

\section{Space time frequency block codes}

Also in the orthogonal frequency division multiplexing system performance is disturbed by the frequency changes or frequency offsets. Which will destroyed the orthogonality of the sub carriers and this tend to increase the inter carrier interference. This leads to disturb the system performance. To avoid the inter carrier Interference there is used inter carrier interference self-cancellation scheme can be used. By this method not only Inter carrier interference is removed but the maximum frequency diversity is achieved through the space time frequency block codes.

\section{Combining multiple cells coordinated setting up for downlink}

The inter carrier interference removed by combining multiple cells coordinated setting up for downlink of multiple input multiple output orthogonal frequency division multiplexing cellular systems. Through this method every base station selects lesser level transmission to every cell edge mobile station within enable simple linear inter carrier interference removed at the mobile station. In this way the required base station collaborated with the interfering base station to transmit the tough inter carrier interference signal. The required base station and interfering base station bring together frequency domain user scheduling and transmission level scheduling in rank to maximize the result of inter carrier interference remove at cell edge mobile station. The coordinated frequency domain user scheduling method needs to lower level transmission scheduling. This method used the low level transmission than the full level .which is selected by the multiple cell coordinated frequency domain user scheduling. Through this method the corresponding base station attempted to choose the mobile station which is located at the cell edge points connecting the base station and allot some of the resource block with the identical frequencies to those cell edge mobile stations. By this method also decreased the spectral efficiency loss. The cell edge mobile stations have not capability for full level multiple input multiple output transmission due to their low signal to interference plus noise power ratio. So the base station use the lower level transmission .because the lower level transmission at the base station helps to decrease the number of efficient nosiness signals.

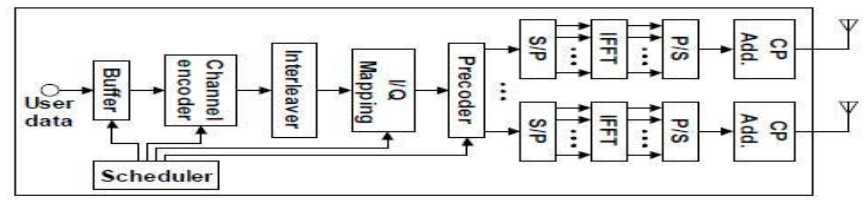

BS TRANSMITTER

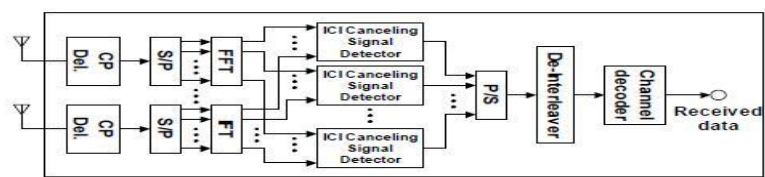

(b) MS TRANSMITTER

Fig1. Block diagram of BS transmitter and MS receiver 


\section{Zero padded symmetric conjugate}

The zero padded symmetric conjugate self-cancellation method is used to remove the ici in the multiple input multiple output -orthogonal frequency division multiplexing system by padding zero between two successive symbols.Inter carrier interference is caused by the frequency changes in the multiple input multiple output system. This method give the benefit of a symmetric conjugate removal and remove the inter carrier interference. Which has been give the diversity gain .The zero padded symmetric conjugate self-cancellation method gain the superior performance than the simple zero padded method in the short pace multiple inputmultiple output-orthogonal frequency division multiplexing for equally AWGN and Rayleigh fading channels in the existence of tiny frequency values changes. This method is used for convenient fading channels. This system offered better CIR than simple one. The system get the low bit error rate in the AWGN channels as compare to the simple zero padded multiple input multiple output-orthogonal frequency division multiplexing system. Rayleigh fading channels offers signal to noise ratio gain of $7.5 \mathrm{db}$ at bit error rate of $10^{\wedge}-4$ in the presence of tiny frequency change of $1-10 \%$.

\section{Complex optimum block adaptive method}

The inter carrier interference in the multiuser multiple input multiple output-orthogonal frequency division multiplexing system has been work in the time varying frequency selective channel environment .Basically inter carrier interference caused by the carrier frequency offsets between the local oscillators in the transmitter and the receiver .which leads to effect on the performance e of the system. By this method the component analysis technique which known as the complex optimum block adaptive method is used to get better the user signal when the inter carrier interference is present .This method exhibited the convergence speed and highly effective to reduce the inter carrier interference by testing the distinct value of signal to noise ratio and carrier frequency offsets and channel time varying parameters.

\section{Parallel interference cancellation}

During the designing of new iterative channel estimation in multiple input and multiple output orthogonal frequency division multiplexing effected by the inter carrier interference. This has been removed by the way of parallel interference cancellation. This is couple with the decision statistical combining. Through this method the data symbol detection should be improved which helped in the channel estimation. When all channel coefficients obtained then inter carrier interference occurred due to the Doppler spread which is minimized by the parallel interference cancellation at the receiver side. After that then the output which is obtained by the parallel interference is then send to the decision statistical combining at that point the decision statistic signal is form and the data symbols are estimated by the detector and the channel estimation should be done without inter carrier interference. Parallel interference cancellation and the decision statistical combining are the better method to remove the inter carrier interference and which enhanced the performance of the channel estimation. The wireless channel is estimated by the Doppler spread and the pilot symbols and the data symbols at the receiver end. In this the every time domain channel coefficient named as the weighted interpolation between the two chosen time domain channel coefficients which is named as the time domain markers. These two time domain markers are chosen in a that way which they have the more correlation with their own channel coefficient. The interpolation weight is basically designed by the Doppler spread information at the receiver side. The data estimation and pilot's symbols to improve the iterative channel estimation.

\section{Curve fitting or weighting factor}

Orthogonal frequency-division multiplexing (OFDM) technique for multiple-input multiple output (MIMO) channels proposed to reduce inter channel interference (ICI) caused by high-speed mobiles in cellular environments. The ICI caused by high-speed mobile channels using a simple curve fitting technique, the weighting factor for group transmission is optimized. Then, a new MIMO-OFDM technique, based on the weighting factor optimization proposed for $\mathrm{y} 35$ reducing ICI caused by time varying channels. Performances of that technique is verified by using the I-METRA channel, proposed for an MIMO channel to 3GPP, and an MIMO-OFDM simulator designed for macro cellular mobile communication. It has shown by computer simulation that the proposed MIMO-OFDM technique has been effective in reducing ICI and noise as well as in obtained diversity gain even under highly-correlated fast fading channels, compared with the conventional MIMO-OFDM schemes.

\section{Turbo equalization algorithm}

To remove the inter carrier interference there has been established new technique name as the turbo equalization algorithm which also used to improve the performance of the MIMO-OFDM system and to improve the channel accuracies of the channel matrix with the iterative channel estimation using the soft decided signal and the pilots symbols. The maximum posterior algorithm MMSE used .In this technique there 
are soft signal or soft information is used for the better performance and removed the inter carrier interference. but the only CSI is accurate and haven't made a thought to renew the channel estimation by using iterative soft decided signal. Any other estimation algorithm for time varying channels in MIMO-OFDM systems is not complete, because the parameters of MIMO channels are always beyond the number of pilots even if the whole MIMO-OFDM signal is regarded as pilot. A low-complexity iterative

equalization for SISO-OFDM systems is proposed based on reducing the ICI lengths to the diagonal band, but ICI shorten windows will not be diagonal band in MIMO-OFDM systems considering the antenna interference, therefore, this method shouldn't applied in MIMO-OFDM systems. Other MAP equalization have been proposed to compensate the influence of the time-varying channel, since it could improve the system performance combined with error correcting code, a MMSE block Turbo iterative equalization is proposed which could improve the BER of better.

\section{Iterative joint estimation}

The use of iterative joint estimation procedure successively canceled the interferences located in the preamble of the OFDM frame. This is used for the joint estimation and initially contained the interferences due to a CP shorter than the channel length. The iterative joint estimation procedure required the steps for proper iterative interference termination algorithm. Which obtained by the initial FO compensation and channel estimation. After the iterative cancellation of interferences, the process performs an additional joint channel and FO estimation whose mean square error converged to the Cramer Rao bound. After this joint estimation permitted the removal of the interferences in the data part of the frame. Which are also due to an insufficient CP in the same iterative fashion but saved iterations compared with the use of other estimation strategies? The evaluation of the procedure has been performed by assessing the convergence of the simulated estimators to the Cramer rao bound as a function of the number of iterations. Iterative joint estimation procedure also improved the performance of the system. It is accomplished that with a reduced number of iterations in the preamble the iterative joint estimation procedure converges to the theoretical bounds reduced the disturbances caused by a hard wireless channel or a purposely inadequate cyclic prefix. The interference cancellation in the data part leads to improvement in the bit error rate and tend the sufficient cyclic prefix.

\section{Orthogonal space time block code}

Inter carrier interference removed by orthogonal space-time block-code in the multiple input multiple output-orthogonal frequency division multiplexing. Through this method the transmitter didn't need to have the frequency offsets and there effect on the bit error rate very less. For spatially correlated multiple input multiple output channels the precoder and Orthogonal space time block code - orthogonal frequency division multiplexing performed with an insignificant bit error rate performance loss. The frequency offsets do not necessary have to be fed back to the transmitter, this reduced the feedback load in closed-loop multiple input multiple output -orthogonal frequency division multiplexing systems and avoid the detrimental effect of frequency-offset mismatched due to imperfect feedback.

\section{Time domain filtering}

To mitigate the inter carrier interference in the multiple input multiple output orthogonal frequency division multiplexing. Analyze the effect of time-variation with in a transmission block which could arise both from Doppler spread of the channel and from synchronization errors. Time domain approach to mitigate the effects of such time-variations. This approach reduced to the familiar single-tap frequency domain equalizer when the channel is block time invariant And also developed this in the context of multiple transmit and receive antennas and specialize the receiver to space-time block-coded systems. Such impairment caused by timevariation within a transmission block and occurs in practice due to both Doppler spread of the transmission channel and due to synchronization errors. Another time-domain filtering-based inter carrier interference mitigation technique which cascaded the time-varying channel with a receive filter so that the overall channel is approximately in the appropriate MSE metric time invariant.

\section{Minimum mean square error equalization}

New minimum mean-square error equalization and ordering process is proposed for multiple input multiple output system to remove the inter carrier interference .during the cyclic prefix reconstruction procedure. This is caused when the cyclic prefix should be less than the length of channel impulse response which has given the results in a loss of the bandwidth efficiency. In this technique a cyclic prefix is added at the head of each symbol and the length of cyclic prefix greater than the expected length of channel impulse response .The another per-tone equalization Space time block coded and cyclic prefix reconstruction technique is applied to a multiple input multiple output-orthogonal frequency division multiplexing system with insufficient cyclic prefix. 


\section{Conclusion}

In this review paper studies about the techniques which are used to remove the inter carrier interference in the multiple input multiple output - orthogonal frequency division multiplexing system inter carrier interference arise in the wireless communication system due to the frequency offsets ,cyclic prefix length. Which is removed by the spatial diversities techniques and the carrier frequency oscillator by measured the other parameters. The best way to mitigate the inter carrier interference in the channel estimation with the technique of parallel interference cancellation and the decision statistically combining at the receiver end. Which improved the performance of the system. The future work should be done on the to mitigate the inter carrier interference by using the decision feedback equalizer.

\section{References}

[1] Xianatao Sun, Qi Wang, Leonard J.Cimini, Larry J. Greenstein and Douglas S. Chan "ICI-Aware Beamforming For MIMO-OFDM Wireless Systems"Member IEEE,vol.11,no.1,january 2012.

[2] Azlina Idris, Norhayati Abdullah, Darmawaty Mohd Ali and Hanis Adiba Mohamad Faculty Of Electrical Engineering,University Teknologi MARA,40450 Shah Alam, Selangor,Malaysia "Evaluation of Pair-Wise Error Probability Performance Using Quarter Subcarrier Mapping Technique in MIMO-OFDM System”, faculty of Electrical Engineering ,University, Teknologi MARA, 40450 Shah Alam Selangor, Malaysia,2013.

[3] Manabu Mikami and Teruya Fujji Wireless System R\&D Center, Softbank Mobile Corp.2-5-10 Aomi, Koto-ku,Tokyo, 135-8070 Japan "An Inter-Cell Interference Cancellation Scheme With Multi-Cell Coordinated Scheduling for Downlink of MIMO/OFDM Cellular Systems" Wireless system R\&D Center, Softbank Mobile Corp.2-5-10 Aomi Kotoku, Tokyo,135-8070 Japan, 2008.

[4] Aurupong Yiwleak, Chaiyod Pirak, and Rudolf Mathar "Zero Padded Symmetric conjugate Technique for Intercarrier Interference Cancellation in MIMO-OFDM Systems.

[5] Raghuram Ranganathan, Thomas Yang and Wasfy Mikhael University of Central Florida, Orlando Florida USA, Embry-Riddle Aeronautical University Daytona Beach, Florida USA "ICI mitigation Multi-User Detection Employing Adaptive ICA MIMOOFDM Systems Time Varient Channels" IEEE, University of Central Florida, Orlando Florida USA, Embry-Riddle Aeronautical University Daytona Beach, Florida USA,2013.

[6] Neda Aboutorab Wibowo Hardjawana and Branka Vucetic Telecommunication Labs, School of Electrical and Information Technology, University of Sydney " Channel Estimation and ICI Cancellation for High Mobility Pilot-Aided MIMO-OFDM Systems", Labs, School of Electrical and Information Technology.

[7] S.G.Haggmen "intercarrier interference self-cancellation scheme for OFDM mobile communication system ".IEEE trans comm...vol.49.pp.1185-1191,july 2001.

[8] Gong Guoqiang and Xia Ping School of Computer and Information Three Gorges University of China, CTGU Yichang, China "Iterative Channel Estimation and Turbo Equalization Using ICI Cancellation for MIMO-OFDM Systems", IEEE, School of Computer and Information Three Gorges University of China, CTGU Yichang ,China, 2011.

[9] Carlos Prieto del Amo and M. Julia Fernández-Getino García, Member,IEEE Transactions on vehicular technology vol.62,no.8,October 2013 "Iterative Joint Estimation Procedure for Channel and Frequency Offset in Multi-Antenna OFDM Systems With an Insufficient Cyclic Prefix", IEEE Transactions on vehicular technology vol.62,no.8, october 2013.

[10] Yu Fu,Chintha Tellambura, Senior Member, IEEE, and Witold A. Krzymie'n senior member IEEE "Transmitter Precoding for ICI Reduction in Closed-Loop MIMO OFDM Systems", vol.56,no.1,january 2007.

[11] Suhas Diggavi and Naofal Al-Dhahir, Anastasios Stamoulis AT\&T Shannon Laboratory, Florham Park, NJ "Intercarrier Interference in MIMO- OFDM", IEEE, AT\&T Shanon Laboratory, Florham Park, 2011.

[12] Jong-Bu Lim, Student Member, IEEE, Chan-Ho Choi, and Gi-Hong Im, Senior Member, "MIMO-OFDM with Insufficient Cyclic Prefix", IEEE, vol.10, no.5,may 2006. 